\title{
THE BYCATCH COMPOSITION OF SHRIMP TRAWL FISHERY IN THE OMAN COASTAL WATERS, THE ARABIAN SEA
}

\author{
Juma M. AL-MAMRY ${ }^{1}$, Mikhail V. CHESALIN²*, Dawood S. AL-MAMARY ${ }^{1}$, \\ and Rashid H. AL-SENAIDI ${ }^{1}$ \\ ${ }^{1}$ Marine Science and Fisheries Centre, Ministry of Agriculture and Fisheries Wealth, \\ Muscat, Sultanate of Oman
}

${ }^{2}$ Fisheries Research Center, Ministry of Agriculture and Fisheries Wealth,

Salalah, Sultanate of Oman

\begin{abstract}
Al-Mamry J.M., Chesalin M.V., Al-Mamary D.S., Al-Senaidi R.H. 2015. The bycatch composition of shrimp trawl fishery in the Oman coastal waters, the Arabian Sea. Acta Ichthyol. Piscat. 45 (3): 273-283.
\end{abstract}

Background. Shrimps are one of the most valuable marine resources of Oman and shrimp fisheries is greatly contributing to the national economy, and sustainability of traditional fisheries villages. However, the shrimp trawl fishery has one of the highest bycatch rate of any fishery and cause negative impacts to marine populations and benthic habitats. This study provides the first data on the composition and abundance of the bycatch in the main shrimp fishing grounds distributed along the south-eastern coastline of Oman in the Arabian Sea.

Materials and methods. Shrimp trawl survey was conducted monthly from January to December 2013 in seven sites using demersal otter trawl. Overall, 93 trawl catches were used for the analysis of the bycatch composition based on weight, number, and frequency of occurrence. Top ten species and top fish families, cephalopods, and crabs were defined in the bycatch. The bycatch compositions were compared in different regions using biodiversity indices and the cluster analysis.

Results. Mean monthly bycatch during the shrimp trawling survey in the coastal waters of Oman in 2013 ranged from 22.2 to $191.5 \mathrm{~kg} \cdot \mathrm{h}^{-1}$, in different months with an overall mean $( \pm \mathrm{SE})$ of $58.7 \pm 5.2 \mathrm{~kg} \cdot \mathrm{h}^{-1}(n=93)$. The bycatch to catch ratio was estimated $8.9: 1$ for shrimp fisheries. This ratio fluctuated significantly over the year with minimum values from April to September. The bycatch composition included 97 bony fish species from 36 families, 12 species from 7 elasmobranchs families and several species of cephalopods and crabs. Catfishes (Ariidae), frogfish (Antennariidae), ponyfishes (Leiognathidae), and rays (Dasyatidae and Gymnuridae), represented a majority of the bycatch.

Conclusion. Shrimp fishery in Oman is affecting mainly small-sized and slow-moving fishes, and invertebrates with low economic value. However, the fine-meshed shrimp trawls also capture juveniles of commercial important fishes and cause some ecological problems. The results from the study point out the necessity of management actions to develop a special policy on bycatch reduction in Oman fisheries.

Keywords: fish, shrimp fisheries, otter trawl, bycatch, Indian Ocean

\section{INTRODUCTION}

Shrimps are one of the most commercially important shellfish resources of Oman greatly contributing to the national economy, consumed on the local market as valuable seafood, and considered an important export commodity. The shrimp fishery plays an important role in rural employment and sustainability of the traditional way of life of Omani people in fisheries villages.

The shrimps are actually harvested in the coastal areas of Al-Wusta, south Al-Sharqiyah and the northern part of
Dhofar provinces. Al-Wusta alone contributes approximately $99 \%$ of the total shrimp landings, in which Mahout (Masirah Gulf) is the most productive area. According to fisheries statistics (Anonymous 2014), in 2010, nearly $854 t$ were landed and $790 t$ of which were exported, earning more than $\$ 5$ million. In 2011, the catch was $952 \mathrm{t}$ and it decreased to $740 \mathrm{t}$ in 2013. To prevent overexploitation of the shrimp and to provide more time for spawning and subsequent growth of the species, the Ministry of Agriculture and Fisheries Wealth shortened the harvest season

* Correspondence: Dr. Mikhail Chesalin, Fisheries Research Center, PC 217, P.O. Box 33, Salalah, Sultanate of Oman, phone: +968 99245605, fax: +968 23219275, e-mail: (MC) mchesalin@ukr.net, (JMAM)drjumabar@hotmail.com, (DSAM)almaamari007@hotmail.com, (RHAS)al-senaidi10@hotmail.com 
from seven months (September-March) to three months (September-November) in 2013.

According to fishery legislation, shrimp fishing is limited to Omani fishers operating small fiberglass boats and using only fishing nets, in addition, all fishers must return to the sea any living small fish that may be found in their catch of shrimp. Historically, traditional fishers use hand casting nets, lately however, they started using bottom otter board trawl nets targeting shrimp in the region. The number of fishing boats, operating in the shrimp fishery, was 378 in Al-Wusta and 18 in Al-Sharqiyah in September 2013 (Anonymous 2014).

Bycatch is generally regarded as a major issue in worldwide fisheries. Shrimp trawl fisheries, particularly for tropical species, were found to generate more discards than any other fishery type and account for just over onethird of the global total (Alverson et al 1994). Aggregate discards from the shrimp fisheries (Saudi Arabia, Kuwait, Iran, Bahrain, and Pakistan) total approximately 100000 $\mathrm{t}$ (Kelleher 2005). In the northwest Indian Ocean, bycatch has been reported for the Pakistani shrimp trawl fishery (Shakir and Bano 1999), Indian shrimp fisheries (Gibinkumar et al. 2012, Mahesh et al. 2014), Iranian shrimp fisheries (Paighambari and Daliri 2012, Kazemi et al. 2013), and Kuwaiti shrimp trawl fishery (Chen et al. 2013), no information, however, has been published concerning bycatch in the shrimp fishery in Oman. This study is the first attempt to analyse the bycatch composition, define the main species, and tendencies in their spatial-temporal distribution of the occurrence, and provides assessments of bycatch rate for Oman's shrimp trawl fishery operating in the Arabian Sea.

\section{MATERIAL AND METHODS}

Sampling operations were carried out in seven shrimp fishing sites: Ras Markaz, Duqm, Khaluf, Attinah, Alreesh, Al-Godn (Al-Wusta region), and Shanaah (Al-Sharqiyah region) throughout January-December in 2013 (Fig. 1). Usually, three sites were sampled each month and three replicate trawls were undertaken in each site (nine trawls in all each month). Overall, 93 trawl catches were used for the analysis of the bycatch composition.

The casting net, which traditionally used to catch shrimps in the region, initially was included as a sampling gear for the survey, however it was impossible to use due to lack of obvious shrimp schools in the shallow waters, therefore, only a small-scale otter trawl was used in this survey. This demersal otter trawl is a cone-shaped net of total length $8.5 \mathrm{~m}$, mouth width $12 \mathrm{~m}$ and height $2.8 \mathrm{~m}$ when open. Mesh sizes in the trawl net and belly were $20 \mathrm{~mm}$ and $10 \mathrm{~mm}$ at the codend. Two beams, floats, and weights maintain the horizontal opening of the trawl while it is towed. This trawl is designed for a shallow water fishery at depth between 3-12 m only. The trawl was towed with a fiberglass fishing boat of $7.6 \mathrm{~m}$ length and $1.2 \mathrm{~m}$ width, powered by one or two outboard engines of $56 \mathrm{~kW}$ (75 HP). The tow duration during the survey was $30 \mathrm{~min}$ with tow speed ranging between $5.6-6.5 \mathrm{~km} \cdot \mathrm{h}^{-1}(3.0-3.5$ knots).

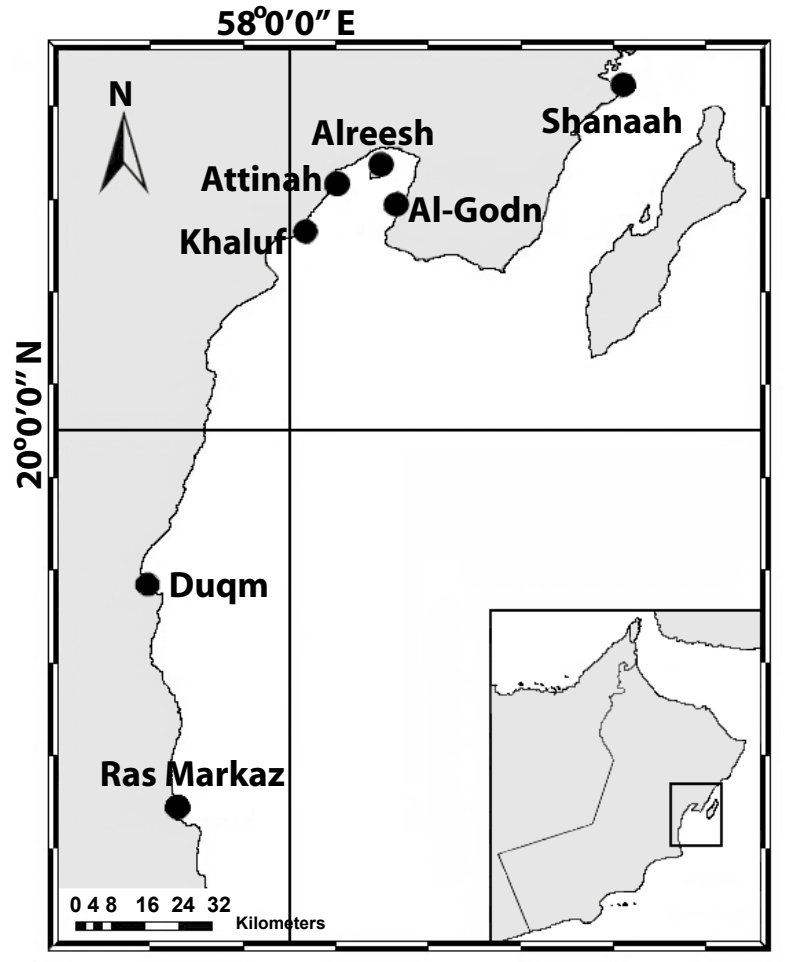

Fig. 1. Sampling sites to study bycatch composition of the shrimp fishery in Oman

After unloading of the total catch on land, it was placed into cool boxes for further analysis in the laboratory. If any rays or turtles encountered in the bycatch, they were immediately measured and returned alive into the sea. The same methodology was followed throughout the three operations in the study site during that day. At the lab, target shrimp and bycatch species were sorted and identified using identification guides (Fischer and Bianchi 1984, Al-Abdessalaam 1995, Randall 1995). Scientific fish names follow FishBase (Froese and Pauly 2015). The fishes and invertebrates were counted, weighed, and minimum and maximum length was measured in each sample. Dominant bycatch families and species were calculated based on weight, number, and frequency of occurrence.

To compare the bycatch composition and abundance in different areas, the diversity indices were estimated and the cluster analysis was conducted using BioDiversity Pro software

\section{RESULTS}

Three penaeid shrimp species, which are targeted by the fishery, were found in these trawl catches: Green tiger prawn, Penaeus semisulcatus, Indian white prawn, Penaeus indicus, and Peregrine shrimp Metapenaeus stebbingi.

The data on total catches of the shrimps and bycatch species during the survey are summarized in Table 1 . The total shrimp catch from 93 trawls was $305.6 \mathrm{~kg}$, and the mean catch ( \pm standard error of the mean) per trawl per hour was $6.6 \pm 1.41 \mathrm{~kg} \cdot \mathrm{h}^{-1}$. The total bycatch was $2728.3 \mathrm{~kg}$, the catches ranged from 22.2 to $191.5 \mathrm{~kg} \cdot \mathrm{h}^{-1}$, and the overall mean was $58.7 \pm 5.2 \mathrm{~kg} \cdot \mathrm{h}^{-1}$. The mean number of bycatch 
animals in a trawl was estimated as $682.4 \pm 37.7$ ind. $\cdot \mathrm{h}^{-1}$. The overall contribution of the shrimps and bycatch species in the total catch was $10.1 \%$ and $89.9 \%$, respectively. The bycatch to shrimp catch ratio $(B: S)$ from the trawling shrimp fishery in Oman was estimated at $8.9: 1$.

The bycatch species dominated in all cases, although the catches and the ratio are notably fluctuated throughout the year. Higher shrimp catches were observed from April to September during the transition and southwest monsoon seasons, with the highest mean values of $34.5 \pm$ $5.8 \mathrm{~kg} \cdot \mathrm{h}^{-1}$ and $24.0 \pm 6.8 \mathrm{~kg} \cdot \mathrm{h}^{-1}$ in May and June, respectively (Table 1). The bycatch was higher $(43.2-191.5 \mathrm{~kg}$. $\mathrm{h}^{-1}$ ) from November to May during the northeast monsoon and was the lowest $\left(22.2-35.9 \mathrm{~kg} \cdot \mathrm{h}^{-1}\right)$ from August to October. However, the bycatch to shrimp catch ratio had slightly different trend. The $B: S$ ratio was low (1.4-6.4 : 1) from April to September. During the open shrimp-fishing season (September-November), the bycatch sharply increased and in November the $B: S$ ratio reached 104.1: 1 , the maximum 117.4 : 1 was observed in January.

The bycatch in the shrimp fishery in Oman is characterized by high diversity and usually consists of a variety of marine species of finfish, squids and crabs. The number of species and groups (crabs were not identified to a species level), occurred during the sampling in a bycatch, ranged from 8 to 32, on the average 20 fish species and some species of crabs and squids were found in each catch. The portion of the main groups in total weight was estimated as follows: bony fishes (Osteichthyes) $70.2 \%$, cartilaginous fishes (Chondrichthyes) $26.4 \%$, crabs (Malacostraca) $1.7 \%$, squids and cuttlefishes (Cephalopoda) $1.5 \%$, other invertebrates $0.2 \%$.

The taxonomic composition of the bycatch species is presented in Table 2. The composition included 97 bony fishes from 36 families, 12 elasmobranch species from 7 families, squid (Sepioteuthis lessoniana), cuttlefish (Sepia pharaonis), and several unidentified species of crabs. From the bony fishes, the most diverse families with the highest number of species were Carangidae (9), followed by Haemulidae (8), Sciaenidae (6), and Clupeidae (5). From the elasmobranchs, Dasyatidae had the majority of species (4), followed by Rhinobatidae (2) and Torpedinidae (2). Two species of shark, Carcharhinus melanopterus (Quoy et Gaimard, 1824) and Stegostoma fasciatum (Hermann, 1783), occurred in the catches. The 13 families of bony fishes and 3 families of elasmobranchs were represented by one species.

The bulk of the bycatch in weight constituted three families of bony fishes (Antennariidae, Ariidae, Leiognathidae) and two ray families (Gymnuridae and Dasyatidae), that represented together $54.7 \%$ of the total catch (Table 3). Same three families of bony fishes (Leiognathidae, Ariidae, and Antennariidae) were also more abundant, accounting for $63.0 \%$ of the total catch in number of individuals. The species from these bony fish families were recorded in $80.6 \%, 63.4 \%$, and $21.5 \%$ of trawl tows year round.

Top ten fish species in the bycatch in weight and number are shown in Table 4. Only four species dominated in the catches in weight, such as longtail butterfly ray, Gymnura poecilura (Shaw, 1804) (Gymnuridae); scarlet frogfish, Antennarius coccineus (Lesson, 1831) (Antennariidae); thinspine sea catfish, Plicofollis tenuispinis (Day, 1877) (Ariidae); and orangefin ponyfish, Photopectoralis bindus (Valenciennes, 1835) (Leiognathidae). The major part of the capture in numbers constituted ponyfishes: Aurigequula fasciata (Lacepède, 1803); Leiognathus oblongus (Valenciennes, 1835); and P. bindus; frogfish, A. coccineus; and catfishes: P. tenuispinis and Netuma thalassina (Rüppell, 1837).

Total $(\mathrm{kg})$ and relative (percentage and $\mathrm{kg} \cdot \mathrm{h}^{-1}$ ) catches of shrimps and bycatch species

Table 1 in Oman coastal waters during shrimp trawl survey 2013

\begin{tabular}{|c|c|c|c|c|c|c|c|c|c|c|c|}
\hline \multirow{2}{*}{ Month } & \multirow{2}{*}{$\begin{array}{l}\text { Number } \\
\text { of trawls }\end{array}$} & \multirow{2}{*}{$\begin{array}{c}\begin{array}{c}\text { Total } \\
\text { catch }\end{array} \\
{[\mathrm{kg}]}\end{array}$} & \multicolumn{4}{|c|}{ Shrimp catch } & \multicolumn{4}{|c|}{ Bycatch } & \multirow{2}{*}{$\begin{array}{l}B: S \\
\text { ratio }\end{array}$} \\
\hline & & & {$[\mathrm{kg}]$} & {$[\%]$} & {$\left[\mathrm{kg} \cdot \mathrm{h}^{-1}\right]$} & $\pm \mathrm{SE}$ & {$[\mathrm{kg}]$} & {$[\%]$} & {$\left[\mathrm{kg} \cdot \mathrm{h}^{-1}\right]$} & $\pm \mathrm{SE}$ & \\
\hline January & 7 & 298.1 & 2.5 & 0.8 & 0.7 & 1.2 & 295.6 & 99.2 & 84.5 & 23.2 & 117.4 \\
\hline February & 4 & 386.7 & 3.7 & 1.0 & 1.8 & 1.4 & 383.0 & 99.0 & 191.5 & 54.3 & 103.7 \\
\hline March & 9 & 302.4 & 12.8 & 4.2 & 2.8 & 1.9 & 289.6 & 95.8 & 64.4 & 12.6 & 22.6 \\
\hline April & 8 & 200.0 & 27.4 & 13.7 & 6.9 & 2.1 & 172.6 & 86.3 & 43.2 & 18.7 & 6.3 \\
\hline May & 3 & 305.8 & 51.7 & 16.9 & 34.5 & 5.8 & 254.1 & 83.1 & 169.4 & 39.4 & 4.9 \\
\hline June & 9 & 264.4 & 108.2 & 40.9 & 24.0 & 6.8 & 156.1 & 59.1 & 34.7 & 14.4 & 1.4 \\
\hline July & 9 & 190.3 & 27.8 & 14.6 & 6.2 & 4.2 & 162.5 & 85.4 & 36.1 & 7.8 & 5.8 \\
\hline August & 9 & 140.9 & 41.2 & 29.2 & 9.1 & 2.3 & 99.7 & 70.8 & 22.2 & 10.9 & 2.4 \\
\hline September & 9 & 120.5 & 16.3 & 13.5 & 3.6 & 3.3 & 104.3 & 86.5 & 23.2 & 6.6 & 6.4 \\
\hline October & 9 & 166.7 & 5.1 & 3.1 & 1.1 & 1.2 & 161.6 & 96.9 & 35.9 & 5.8 & 31.7 \\
\hline November & 9 & 410.0 & 3.9 & 1.0 & 0.9 & 0.6 & 406.1 & 99.0 & 90.2 & 26.5 & 104.1 \\
\hline December & 8 & 248.0 & 5.0 & 2.0 & 1.3 & 0.8 & 243.0 & 98.0 & 60.7 & 14.7 & 48.5 \\
\hline Total & 93 & 3033.9 & 305.6 & 10.1 & 6.6 & 2.2 & 2728.3 & 89.9 & 58.7 & 31.2 & 8.9 \\
\hline
\end{tabular}

$B: S$ ratio = bycatch to shrimp catch ratio. 
Table 2

The composition of bycatch species during shrimp trawl survey in Oman coastal waters of the Arabian Sea in 2013

\begin{tabular}{|c|c|c|c|c|c|c|c|c|}
\hline \multirow[t]{2}{*}{ Family } & \multirow[t]{2}{*}{ Species } & \multicolumn{2}{|c|}{$\begin{array}{l}\text { No. of } \\
\text { records }\end{array}$} & \multicolumn{2}{|c|}{$\begin{array}{c}\text { Total weight } \\
\text { caught }\end{array}$} & \multicolumn{2}{|c|}{$\begin{array}{c}\text { Total number } \\
\text { caught }\end{array}$} & \multirow{2}{*}{$\begin{array}{c}\text { Length } \\
\text { range } \\
{[\mathrm{cm}]}\end{array}$} \\
\hline & & $n$ & {$[\%]$} & {$[\mathrm{kg}]$} & {$[\%]$} & $n$ & {$[\%]$} & \\
\hline Carcharhinidae & Carcharhinus melanopterus (Quoy et Gaimard, 1824) & 9 & 9.7 & 3.87 & 0.21 & 21 & 0.06 & $11-50$ \\
\hline Stegostomatidae & Stegostoma fasciatum (Hermann, 1783) & 1 & 1.1 & 0.08 & 0.00 & 1 & 0.00 & 29 \\
\hline \multirow[t]{5}{*}{ Dasyatidae } & & 63 & 67.7 & 212.16 & 11.15 & 203 & 0.62 & \\
\hline & Himantura gerrardi (Gray, 1851) & 1 & 1.1 & 0.65 & 0.03 & 1 & 0.00 & 28 \\
\hline & Himantura imbricata (Bloch et Schneider, 1801) & 49 & 52.7 & 29.70 & 1.56 & 160 & 0.49 & $4-29$ \\
\hline & Himantura uarnak (Gmelin, 1789) & 13 & 14.0 & 168.90 & 8.87 & 14 & 0.04 & $18-120$ \\
\hline & Taeniura lymma (Forsskål, 1775) & 10 & 10.8 & 5.86 & 0.31 & 23 & 0.07 & $8-31$ \\
\hline Gymnuridae & Gymnura poecilura (Shaw, 1804) & 60 & 64.5 & 308.351 & 14.30 & 256 & 0.78 & $7-103$ \\
\hline Myliobatidae & Rhinoptera jayakari Boulenger, 1895 & 2 & 2.2 & 3.864 & 0.20 & 2 & 0.01 & $32-34$ \\
\hline \multirow[t]{3}{*}{ Rhinobatidae } & & 21 & 22.6 & 12.218 & 0.64 & 46 & 0.14 & \\
\hline & Glaucostegus granulatus (Cuvier, 1829) & 20 & 21.5 & 12.0692 & 0.63 & 44 & 0.13 & $15-98$ \\
\hline & Rhinobatos salalah Randall et Compagno, 1995 & 1 & 1.1 & 0.149 & 0.01 & 2 & 0.01 & $28-32$ \\
\hline \multirow[t]{3}{*}{ Torpedinidae } & & 15 & 16.1 & 26.187 & 1.38 & 21 & 0.06 & \\
\hline & Torpedo panthera Olfers, 1831 & 13 & 14.0 & 26.166 & 1.37 & 19 & 0.06 & $9-91$ \\
\hline & Torpedo sinuspersici Olfers, 1831 & 2 & 2.2 & 0.021 & 0.00 & 2 & 0.01 & $10-11$ \\
\hline Antennariidae & Antennarius coccineus (Lesson, 1831) & 20 & 21.5 & 214.487 & 11.27 & 4975 & 15.23 & $5-43$ \\
\hline Apistidae & Apistus carinatus (Bloch et Schneider, 1801) & 6 & 6.5 & 4.696 & 0.25 & 148 & 0.45 & $6-13$ \\
\hline \multirow[t]{3}{*}{ Apogonidae } & & 4 & 4.3 & 0.346 & 0.02 & 29 & 0.09 & \\
\hline & Apogonichthyoides nigripinnis (Cuvier, 1828) & 3 & 3.2 & 0.083 & 0.00 & 16 & 0.05 & $7-9$ \\
\hline & Ostorhinchus gularis (Fraser et Lachner, 1984) & 4 & 4.3 & 0.263 & 0.01 & 13 & 0.04 & $8-13$ \\
\hline \multirow[t]{5}{*}{ Ariidae } & & 59 & 63.4 & 220.199 & 11.57 & 6277 & 19.21 & \\
\hline & Arius maculatus (Thunberg, 1792) & 4 & 4.3 & 6.271 & 0.33 & 41 & 0.13 & $8-25$ \\
\hline & Netuma bilineata (Valenciennes, 1840) & 4 & 4.3 & 23.58 & 1.24 & 59 & 0.18 & $14-31$ \\
\hline & Netuma thalassina (Rüppell, 1837) & 30 & 32.3 & 100.77 & 5.29 & 3742 & 11.46 & $5-46$ \\
\hline & Plicofollis tenuispinis (Day, 1877) & 23 & 24.7 & 89.58 & 4.70 & 2435 & 7.71 & $6-37$ \\
\hline \multirow[t]{10}{*}{ Carangidae } & & 35 & 37.6 & 19.098 & 1.00 & 328 & 1.00 & \\
\hline & Alepes djedaba (Forsskål, 1775) & 2 & 2.2 & 0.53 & 0.03 & 8 & 0.02 & $7-16$ \\
\hline & Alepes kleinii (Bloch, 1793) & 14 & 15.1 & 4.94 & 0.26 & 164 & 0.50 & $7-15$ \\
\hline & Carangoides armatus (Rüppell, 1830) & 6 & 6.5 & 2.65 & 0.14 & 57 & 0.17 & $9-24$ \\
\hline & Carangoides chrysophrys (Cuvier, 1833) & 1 & 1.1 & 0.1 & 0.01 & 1 & 0.00 & 18 \\
\hline & Carangoides malabaricus (Bloch et Schneider, 1801) & 5 & 5.4 & 0.89 & 0.04 & 11 & 0.04 & $15-24$ \\
\hline & Carangoides praeustus (Anonymous [Bennett], 1830) & 3 & 3.2 & 0.26 & 0.01 & 4 & 0.01 & $15-20$ \\
\hline & Gnathanodon speciosus (Forsskål, 1775) & 1 & 1.1 & 8.0 & 0.42 & 1 & 0.00 & 37 \\
\hline & Scomberoides commersonianus Lacepède, 1801 & 3 & 3.2 & 0.114 & 0.01 & 6 & 0.02 & $12-14$ \\
\hline & Selaroides leptolepis (Cuvier, 1833) & 10 & 10.8 & 2.36 & 0.12 & 72 & 0.22 & $13-17$ \\
\hline \multirow[t]{6}{*}{ Clupeidae } & & 54 & 58.1 & 20.04 & 1.05 & 503 & 1.54 & \\
\hline & Anodontostoma chacunda (Hamilton, 1822) & 3 & 3.2 & 0.74 & 0.04 & 17 & 0.05 & $13-18$ \\
\hline & Hilsa kelee (Cuvier, 1829) & 5 & 5.4 & 3.11 & 0.16 & 14 & 0.04 & $14-33$ \\
\hline & Nematalosa nasus (Bloch, 1795) & 11 & 11.8 & 1.39 & 0.07 & 18 & 0.06 & $8-22$ \\
\hline & Sardinella albella (Valenciennes, 1847) & 50 & 53.8 & 13.92 & 0.73 & 432 & 1.32 & $8-33$ \\
\hline & Sardinella longiceps Valenciennes, 1847 & 6 & 6.5 & 0.89 & 0.05 & 22 & 0.07 & $21-23$ \\
\hline \multirow[t]{5}{*}{ Cynoglossidae } & & 65 & 69.9 & 24.23 & 1.27 & 562 & 1.72 & \\
\hline & Cynoglossus bilineatus (Lacepède, 1802) & 59 & 63.4 & 22.43 & 1.18 & 485 & 1.48 & $5-38$ \\
\hline & Cynoglossus kopsii (Bleeker, 1851) & 10 & 10.8 & 0.36 & 0.02 & 20 & 0.06 & $10-13$ \\
\hline & Cynoglossus lachneri Menon, 1977 & 1 & 1.1 & 0.74 & 0.04 & 4 & 0.01 & $21-27$ \\
\hline & Paraplagusia blochii (Bleeker, 1851) & 10 & 10.8 & 0.704 & 0.04 & 53 & 0.16 & $3-14$ \\
\hline Drepaneidae & Drepane longimana (Bloch et Schneider, 1801) & 16 & 17.2 & 4.92 & 0.26 & 35 & 0.11 & $5-33$ \\
\hline \multirow[t]{3}{*}{ Engraulidae } & & 45 & 48.4 & 7.605 & 0.40 & 436 & 1.33 & \\
\hline & Thryssa baelama (Forsskål, 1775) & 2 & 2.2 & 0.071 & 0.00 & 13 & 0.04 & $10-11$ \\
\hline & Thryssa hamiltonii Gray, 1835 & 43 & 46.2 & 7.534 & 0.40 & 423 & 1.29 & $5-21$ \\
\hline Ephippidae & Platax teira (Forsskål, 1775) & 2 & 2.2 & 0.756 & 0.04 & 2 & 0.01 & $7-30$ \\
\hline
\end{tabular}

Table continues on next page. 


\begin{tabular}{|c|c|c|c|c|c|c|c|c|}
\hline \multirow{2}{*}{ Family } & \multirow{2}{*}{ Species } & \multicolumn{2}{|c|}{$\begin{array}{l}\text { No. of } \\
\text { records }\end{array}$} & \multicolumn{2}{|c|}{$\begin{array}{l}\text { Total weight } \\
\text { caught }\end{array}$} & \multicolumn{2}{|c|}{$\begin{array}{l}\text { Total number } \\
\text { caught }\end{array}$} & \multirow{2}{*}{$\begin{array}{c}\text { Length } \\
\text { range } \\
{[\mathrm{cm}]}\end{array}$} \\
\hline & & $n$ & {$[\%]$} & {$[\mathrm{kg}]$} & {$[\%]$} & $n$ & {$[\%]$} & \\
\hline \multirow[t]{3}{*}{ Gerreidae } & & 56 & 60.2 & 23.145 & 1.22 & 604 & 1.85 & \\
\hline & Gerres filamentosus Cuvier, 1829 & 43 & 46.2 & 15.326 & 0.81 & 409 & 1.25 & $5-22$ \\
\hline & Gerres longirostris (Lacepède, 1801) & 33 & 35.5 & 7.819 & 0.41 & 195 & 0.60 & $10-27$ \\
\hline Gobiidae & Coryogalops anomolus Smith, 1958 & 2 & 2.2 & 0.048 & 0.00 & 4 & 0.01 & $8-10$ \\
\hline \multirow[t]{9}{*}{ Haemulidae } & & 25 & 26.9 & 15.976 & 0.84 & 62 & 0.19 & \\
\hline & Diagramma pictum (Thunberg, 1792) & 1 & 1.1 & 9.6 & 0.50 & 1 & 0.00 & 12 \\
\hline & Plectorhinchus gaterinus (Forsskål, 1775) & 1 & 1.1 & 0.284 & 0.01 & 8 & 0.02 & $11-15$ \\
\hline & Plectorhinchus picus (Cuvier, 1828) & 2 & 2.2 & 0.096 & 0.01 & 2 & 0.01 & 11 \\
\hline & Plectorhinchus schotaf (Forsskål, 1775) & 1 & 1.1 & 0.05 & 0.00 & 2 & 0.01 & $12-13$ \\
\hline & Plectorhinchus sordidus (Klunzinger, 1870) & 2 & 2.2 & 0.038 & 0.00 & 3 & 0.01 & $8-10$ \\
\hline & Pomadasys commersonnii (Lacepède, 1801) & 4 & 4.3 & 3.538 & 0.19 & 5 & 0.02 & $10-16$ \\
\hline & Pomadasys kaakan (Cuvier, 1830) & 18 & 19.4 & 2.3 & 0.12 & 40 & 0.12 & $9-23$ \\
\hline & Pomadasys olivaceus (Day, 1875) & 1 & 1.1 & 0.072 & 0.00 & 1 & 0.00 & 16 \\
\hline \multirow[t]{5}{*}{ Leiognathidae } & & 75 & 80.6 & 191.35 & 10.05 & 11355 & 34.76 & \\
\hline & Aurigequula fasciata (Lacepède, 1803) & 19 & 20.4 & 42.591 & 2.24 & 1956 & 5.99 & $4-14$ \\
\hline & Karalla daura (Cuvier, 1829) & 18 & 19.4 & 8.945 & 0.47 & 443 & 1.36 & $4-16$ \\
\hline & Leiognathus oblongus (Valenciennes, 1835) & 23 & 24.7 & 41.414 & 2.18 & 3793 & 11.61 & $5-15$ \\
\hline & Photopectoralis bindus (Valenciennes, 1835) & 43 & 46.2 & 98.360 & 5.17 & 5163 & 15.80 & $5-26$ \\
\hline Lethrininidae & Lethrinus nebulosus (Forsskål, 1775) & 1 & 1.1 & 0.156 & 0.01 & 3 & 0.01 & 14 \\
\hline \multirow[t]{3}{*}{ Monacanthidae } & & 3 & 3.2 & 0.043 & 0.00 & 4 & 0.01 & \\
\hline & $\begin{array}{l}\text { Paramonacanthus oblongus (Temminck et Schlegel, } \\
\text { 1850) }\end{array}$ & 1 & 1.1 & 0.006 & 0.00 & 1 & 0.00 & 7 \\
\hline & Stephanolepis diaspros Fraser-Brunner, 1940 & 2 & 2.2 & 0.037 & 0.00 & 3 & 0.01 & $8-9$ \\
\hline \multirow[t]{4}{*}{ Mugilidae } & & 5 & 5.4 & 1.104 & 0.06 & 9 & 0.03 & \\
\hline & Chelon subviridis (Valenciennes, 1836) & 2 & 2.2 & 0.704 & 0.04 & 6 & 0.02 & $10-24$ \\
\hline & Liza persicus (Senou, Randall et Okiyama, 1995) & 1 & 1.1 & 0.252 & 0.01 & 1 & 0.00 & 26 \\
\hline & Moolgarda pedaraki (Valenciennes, 1836) & 2 & 2.2 & 0.148 & 0.01 & 2 & 0.00 & $18-19$ \\
\hline \multirow[t]{4}{*}{ Mullidae } & & 39 & 41.9 & 10.452 & 0.55 & 339 & 1.04 & \\
\hline & Parupeneus heptacanthus (Lacepède, 1802) & 3 & 3.2 & 0.068 & 0.00 & 3 & 0.01 & $8-16$ \\
\hline & Upeneus sundaicus (Bleeker, 1855) & 34 & 36.6 & 9.892 & 0.52 & 313 & 0.96 & $6-27$ \\
\hline & Upeneus tragula Richardson, 1846 & 4 & 4.3 & 0.193 & 0.01 & 23 & 0.07 & $5-15$ \\
\hline \multirow[t]{4}{*}{ Nemipteridae } & & 11 & 11.8 & 3.35 & 0.18 & 83 & 0.25 & \\
\hline & Nemipterus japonicus (Bloch, 1791) & 9 & 9.7 & 2.498 & 0.13 & 68 & 0.21 & $7-16$ \\
\hline & Nemipterus peronii (Valenciennes, 1830) & 2 & 2.2 & 0.539 & 0.03 & 10 & 0.03 & $13-23$ \\
\hline & Scolopsis taeniatus (Cuvier, 1830) & 2 & 2.2 & 0.313 & 0.02 & 5 & 0.02 & $14-20$ \\
\hline \multirow[t]{4}{*}{ Paralichthyidae } & & 57 & 61.3 & 15.345 & 0.81 & 470 & 1.44 & \\
\hline & Pseudorhombus arsius (Hamilton, 1822) & 32 & 34.4 & 4.272 & 0.22 & 93 & 0.28 & $7-23$ \\
\hline & Pseudorhombus malayanus Bleeker, 1865 & 35 & 37.6 & 7.468 & 0.39 & 344 & 1.05 & $5-18$ \\
\hline & $\begin{array}{l}\text { Pseudorhombus triocellatus (Bloch et Schneider, } \\
\text { 1801) }\end{array}$ & 17 & 18.3 & 3.605 & 0.19 & 33 & 0.10 & $12-39$ \\
\hline \multirow[t]{4}{*}{ Platycephalidae } & & 35 & 37.6 & 15.388 & 0.81 & 135 & 0.41 & \\
\hline & Grammoplites suppositus (Troschel, 1840) & 10 & 10.8 & 3.112 & 0.16 & 45 & 0.14 & $14-23$ \\
\hline & Platycephalus indicus (Linnaeus, 1758) & 7 & 7.5 & 3.052 & 0.16 & 27 & 0.08 & $7-37$ \\
\hline & Sorsogona tuberculata (Cuvier, 1829) & 21 & 22.6 & 9.224 & 0.48 & 63 & 0.19 & $17-53$ \\
\hline Plotosidae & Plotosus lineatus (Thunberg, 1787) & 30 & 32.3 & 38.006 & 2.00 & 610 & 1.87 & $17-53$ \\
\hline Polynemidae & Polydactylus sextarius (Bloch et Schneider, 1801) & 23 & 24.7 & 32.5 & 1.71 & 224 & 0.69 & $8-63$ \\
\hline Pomatomidae & Pomatomus saltatrix (Linnaeus, 1766) & 2 & 2.2 & 0.304 & 0.02 & 2 & 0.01 & $20-28$ \\
\hline \multirow[t]{7}{*}{ Sciaenidae } & & 51 & 54.8 & 66.362 & 3.49 & 987 & 3.02 & \\
\hline & Johnius belangerii (Cuvier, 1830) & 13 & 14.0 & 1.312 & 0.07 & 57 & 0.17 & $7-17$ \\
\hline & Johnius borneensis (Bleeker, 1851) & 4 & 4.3 & 0.859 & 0.05 & 10 & 0.03 & $14-22$ \\
\hline & Johnius dussumieri (Cuvier, 1830) & 25 & 26.9 & 21.924 & 1.15 & 253 & 0.77 & $6-25$ \\
\hline & Otolithes cuvieri Trewavas, 1974 & 30 & 32.3 & 37.384 & 1.96 & 588 & 1.80 & $6-33$ \\
\hline & Otolithes ruber (Bloch et Schneider, 1801) & 14 & 15.1 & 4.842 & 0.25 & 78 & 0.24 & $6-37$ \\
\hline & Protonibea diacanthus (Lacepède, 1802) & 1 & 1.1 & 0.036 & 0.00 & 1 & 0.00 & 16 \\
\hline
\end{tabular}

Table continues on next page. 
Table 2 cont

\begin{tabular}{|c|c|c|c|c|c|c|c|c|}
\hline \multirow[t]{2}{*}{ Family } & \multirow[t]{2}{*}{ Species } & \multicolumn{2}{|c|}{$\begin{array}{l}\text { No. of } \\
\text { records }\end{array}$} & \multicolumn{2}{|c|}{$\begin{array}{c}\text { Total weight } \\
\text { caught }\end{array}$} & \multicolumn{2}{|c|}{$\begin{array}{c}\text { Total number } \\
\text { caught }\end{array}$} & \multirow{2}{*}{$\begin{array}{c}\text { Length } \\
\text { range } \\
{[\mathrm{cm}]}\end{array}$} \\
\hline & & $n$ & {$[\%]$} & {$[\mathrm{kg}]$} & {$[\%]$} & $n$ & {$[\%]$} & \\
\hline Scorpaenidae & Choridactylus lineatus Poss et Mee, 1995 & 2 & 2.2 & 0.052 & 0.00 & 2 & 0.01 & $10-11$ \\
\hline \multirow[t]{4}{*}{ Sillaginidae } & & 55 & 59.1 & 61.211 & 3.22 & 782 & 2.39 & \\
\hline & Sillago arabica McKay et McCarthy, 1989 & 33 & 35.5 & 30.942 & 1.63 & 429 & 1.31 & $5-25$ \\
\hline & Sillago attenuata McKay, 1985 & 13 & 14.0 & 9.399 & 0.49 & 156 & 0.48 & $6-27$ \\
\hline & Sillago sihama (Forsskål, 1775) & 10 & 10.8 & 20.87 & 1.10 & 197 & 0.60 & $4-25$ \\
\hline \multirow[t]{5}{*}{ Soleidae } & & 52 & 55.9 & 10.495 & 0.55 & 316 & 0.97 & \\
\hline & Brachirus orientalis (Bloch et Schneider, 1801) & 23 & 24.7 & 5.794 & 0.30 & 67 & 0.21 & $7-28$ \\
\hline & Solea elongata Day, 1877 & 36 & 38.7 & 3.585 & 0.19 & 223 & 0.68 & $3-16$ \\
\hline & Solea stanalandi Randall et McCarthy, 1989 & 6 & 6.5 & 0.284 & 0.01 & 23 & 0.07 & $9-19$ \\
\hline & Zebrias captivus Randall, 1995 & 3 & 3.2 & 0.832 & 0.04 & 3 & 0.01 & $26-35$ \\
\hline \multirow[t]{5}{*}{ Sparidae } & & 43 & 46.2 & 24.858 & 1.31 & 190 & 0.58 & \\
\hline & Acanthopagrus latus (Houttuyn, 1782) & 4 & 4.3 & 2.342 & 0.12 & 4 & 0.01 & $11-39$ \\
\hline & Pagellus affinis Boulenger, 1888 & 11 & 11.8 & 5.054 & 0.27 & 100 & 0.31 & $8-20$ \\
\hline & Rhabdosargus haffara (Forsskål, 1775) & 3 & 3.2 & 2.384 & 0.13 & 22 & 0.07 & $13-15$ \\
\hline & Rhabdosargus sarba (Forsskål, 1775) & 32 & 34.4 & 15.078 & 0.79 & 64 & 0.20 & $8-63$ \\
\hline \multirow[t]{3}{*}{ Sphyraenidae } & & 15 & 16.1 & 2.512 & 0.13 & 31 & 0.09 & \\
\hline & Sphyraena obtusata Cuvier, 1829 & 14 & 15.1 & 2.058 & 0.11 & 29 & 0.09 & $15-35$ \\
\hline & Sphyraena putnamiae Jordan et Seale, 1905 & 1 & 1.1 & 0.454 & 0.02 & 2 & 0.01 & $32-46$ \\
\hline Synanceiidae & Minous monodactylus (Bloch et Schneider, 1801) & 3 & 3.2 & 0.047 & 0.00 & 3 & 0.01 & $6-15$ \\
\hline \multirow[t]{3}{*}{ Synodontidae } & & 63 & 67.7 & 74.227 & 3.90 & 705 & 2.16 & \\
\hline & Saurida tumbil (Bloch, 1795) & 47 & 50.5 & 59.598 & 3.13 & 579 & 1.77 & $16-60$ \\
\hline & Saurida undosquamis (Richardson, 1848) & 27 & 29.0 & 14.629 & 0.77 & 126 & 0.39 & $13-37$ \\
\hline \multirow[t]{4}{*}{ Terapontidae } & & 70 & 75.3 & 10.965 & 0.58 & 474 & 1.45 & \\
\hline & Pelates quadrilineatus (Bloch, 1790) & 2 & 2.2 & 0.326 & 0.02 & 7 & 0.02 & $11-23$ \\
\hline & Terapon jarbua (Forsskål, 1775) & 5 & 5.4 & 0.342 & 0.02 & 15 & 0.05 & $9-14$ \\
\hline & Terapon puta Cuvier, 1829 & 67 & 72.0 & 10.297 & 0.54 & 452 & 1.38 & $3-30$ \\
\hline \multirow[t]{4}{*}{ Tetraodontidae } & & 59 & 63.4 & 60.467 & 3.18 & 301 & 0.92 & \\
\hline & Arothron immaculatus (Bloch et Schneider, 1801) & 4 & 4.3 & 2.926 & 0.15 & 4 & 0.01 & $20-37$ \\
\hline & Chelondon patoca (Hamilton, 1822) & 6 & 6.5 & 1.473 & 0.08 & 18 & 0.06 & $6-32$ \\
\hline & Lagocephalus guentheri Miranda Ribeiro, 1915 & 50 & 53.8 & 53.868 & 2.83 & 271 & 0.83 & $4-37$ \\
\hline Triacanthidae & Pseudotriacanthus strigilifer (Cantor, 1849) & 38 & 40.9 & 16.370 & 0.86 & 343 & 1.05 & $3-25$ \\
\hline Trichiuridae & Trichiurus lepturus Linnaeus, 1758 & 3 & 3.2 & 0.189 & 0.01 & 5 & 0.02 & $26-52$ \\
\hline UI fishes & & 69 & 74.2 & 219.583 & 11.54 & 1772 & 5.42 & \\
\hline Loliginidae & Sepioteuthis lessoniana Lesson, 1830 & 35 & 37.6 & 3.66 & 0.19 & 155 & 0.47 & $3-20$ \\
\hline Sepiidae & Sepia pharaonis Ehrenberg, 1831 & 58 & 62.4 & 46.611 & 2.45 & 300 & 0.92 & $2-48$ \\
\hline UI crabs & & 64 & 68.8 & 55.56 & 2.92 & 356 & 1.09 & $3-18$ \\
\hline
\end{tabular}

UI fishes $=$ unidentified fishes, UI crabs $=$ unidentified crabs.

Only five species of bony fishes including Terapon puta Cuvier, 1829 (Terapontidae); Cynoglossus bilineatus (Lacepède, 1802) (Cynoglossidae); Sardinella albella (Valenciennes, 1847) (Clupeidae); Lagocephalus guentheri Miranda Ribeiro, 1915 (Tetraodontidae); and Saurida tumbil (Bloch, 1795) (Synodontidae); two rays: Gymnura poecilura (Shaw, 1804) (Gymnuridae) and Himantura imbricata (Bloch et Schneider, 1801) (Dasyatidae); cuttlefish Sepia pharaonis (Sepiidae); and crabs occurred in more than $50 \%$ collected samples.

The richest site in the bycatch diversity (78 species) was Duqm, followed by Al-Godn (76) and Khaluf (68) (Fig. 2A). The lowest number of the bycatch species was registered on the outer sites as Ras Markaz (20) and Shanaah (26).

The higher bycatch rate in weight with means ranged between $60-70 \mathrm{~kg} \cdot \mathrm{h}^{-1}$ was in southern sites of Ras Markaz,
Duqm and Khaluf (Fig. 2A). Notably lower catches was observed in the central area between Attinah, Alreesh and Al-Godn (mean 20-25 kg $\cdot \mathrm{h}^{-1}$ ). The picture of the bycatch rate in numbers is different (Fig. 2B). The highest number of individuals per trawl per hour was registered in Shanaah (1996 ind. $\left.\cdot \mathrm{h}^{-1}\right)$ and the lowest in Attinah (237 ind. $\left.\cdot \mathrm{h}^{-1}\right)$.

Tables 3 and 4 also show differences between studied sites in the bycatch rate (in weight and numbers) of the dominant families and species, respectively. Three most important families sampled in Ras Markaz were Tetraodontidae (31.3\%), Gymnuridae (14.3\%), and Sillaginidae (14.1\%). The dominant species here were Gymnura poecilura, Lagocephalus guentheri, and Sillago sihama (Forsskål, 1775). The frogfish Antennarius coccineus was the most abundant bycatch species in Duqm that contributed $28.4 \%$ in weight and $19.3 \%$ in total number of 

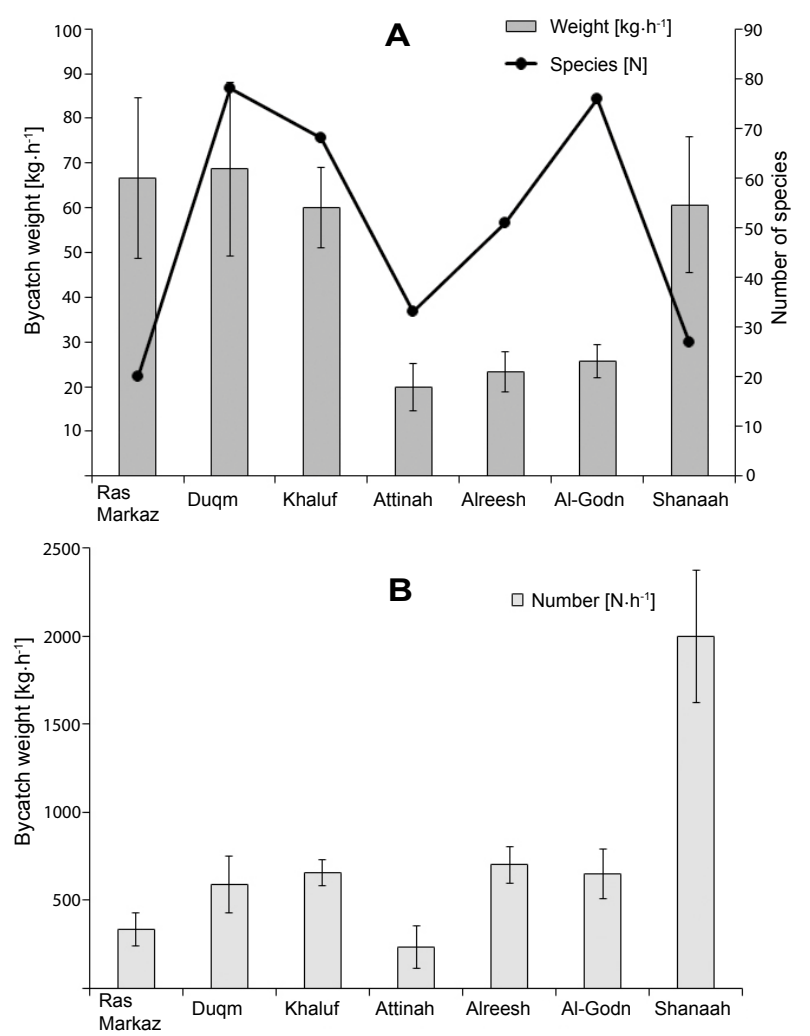

Fig. 2. Some bycatch characteristics in different locations: A - total number of the bycatch species and mean weight of the bycatch $\left(\mathrm{kg} \cdot \mathrm{h}^{-1}\right.$, with SE bars $), \mathbf{B}$ - mean number of bycatch specimens (ind. $\cdot \mathrm{h}^{-1}$, with SE bars)

collected samples. Representatives of rays-G. poecilura and Himantura uarnak (Gmelin, 1789) — took second and third place in weight, while in numbers second dominated fishes was from family Leiognathidae-Aurigequula fasciata (Lacepède, 1803) and Photopectoralis bindus). Similar bycatch composition and structure were observed in Khaluf. The dominant families (in weight) here were presented by rays (Gymnuridae, Dasyatidae) and catfishes (Ariidae), whereas in numbers by ponyfishes (Leiognathidae) and catfishes (Ariidae). The main shrimp fishing ground of Oman is located in Masirah Gulf (Mahout region), therefore the majority of our study sites were concentrated in this region. Three sites (Attinah, Alreesh, and Al-Godn) are located very close to each other (Fig. 1). This region was the richest in the bycatch diversity (78 species and 37 families), but had the lowest rate in relative bycatch weight per sample (Fig. 2). The frogfish $A$. coccineus (Antennariidae) was found the most abundant species in weight and numbers in Attinah, besides significant part of the catches comprised rays ( $G$. poecilura and Himantura imbricata). The dominant families in Alreesh and Al-Godn were Leiognathidae, Ariidae and Gymnuridae and dominant species here was $P$. bindus (Leiognathidae). The Leiognathus oblongus (Leiognathidae) absolutely dominated in catches in Shanaah.

The largest catches of cuttlefish (Sepia pharaonis) were found in southern part of the studied region in Ras Markaz and Duqm. Crabs were more common in Masirah
Gulf (Attinah, Alreesh and Al-Godn). Several specimens of blacktip reef shark (Carcharhinus melanopterus) were caught in Duqm and Khaluf.

Concerning the size composition of the catches, it is worthy to note that rays (Dasyatidae, Gymnuridae, Rhinobatidae, and Torpedinidae) were presented by large-sized specimens (up to $120 \mathrm{~cm}$, mean approximately $30 \mathrm{~cm}$ ), so they constituted a significant portion of the bycatch in weight, however in most cases they occurred rarely and their number was low. Total length of other fishes varied from 2.0 to $54.0 \mathrm{~cm}$ (mean $15.8 \mathrm{~cm}$ ). Such dominant fish as Antennarius coccineus (Antennariidae) had length ranged between 5 and $43 \mathrm{~cm}$ (mean $20.2 \mathrm{~cm}$ ). Mean length of the catfishes (Arius maculatus (Thunberg, 1792); Netuma bilineata (Valenciennes, 1840); Netuma thalassina; and Plicofollis tenuispinis) was $18.0 \mathrm{~cm}$ (range $5-46 \mathrm{~cm}$ ). The length of ponyfishes varied from 4 to $26 \mathrm{~cm}$, mean length was about $10 \mathrm{~cm}$.

According to the calculation of biodiversity indices, the level of bycatch diversity in studied sites was very high and the highest values of Shannon-, Simpson-, and Hill's indices were found in Alreesh and Al-Godh sites, while lower species diversity with few dominant species was more typical for Shanaah (Table 5). However, on the data of the calculation of the Margalef's index of the community diversity, the higher index and the greater diversity was registered in Attinah.

The dendrogram was constructed to express the result of the cluster analysis based on the weight data (Fig. 3). The Bray-Curtis cluster analysis separated the studied sites into six groups. The cluster plot showed a high degree of similarity between Alreesh, Attinah, and Al-Godn, and more similarity of these sites with Duqm and Khaluf, while more differences were displayed between Ras Markaz and Shanaah.

The seasonal changes in composition, weight, and numbers of the bycatch species were observed during the survey. Overall, bony fishes had the higher catches during northeast monsoon period from October to May. Elasmobranchs were more abundant in November-April. On the contrary, cephalopods and crabs have occurred

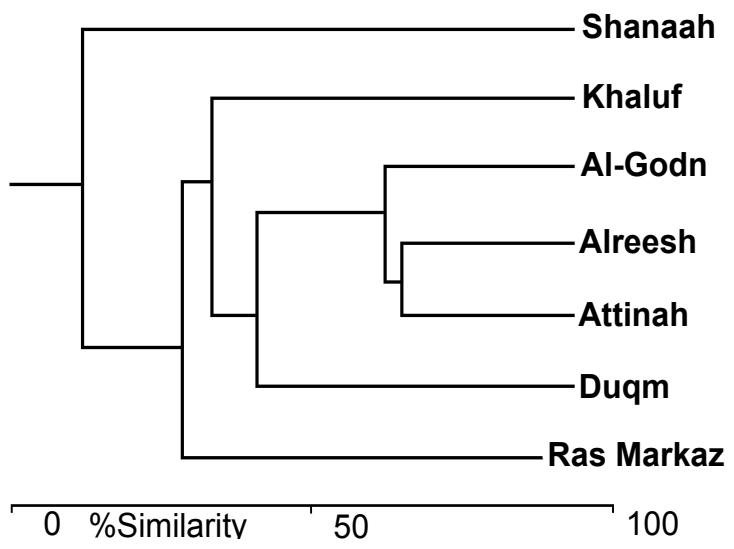

Fig. 3. Dendrogram showing the similarity of the bycatch diversity among studied sites based on weight of species 
Table 3

Top ten fish families, cuttlefish, and crabs in percentages of weight and number in the bycatch during the shrimp trawl survey 2013

\begin{tabular}{|c|c|c|c|c|c|c|c|c|}
\hline \multirow{2}{*}{ Family } & \multicolumn{8}{|c|}{ Site } \\
\hline & Ras Markaz & Duqm & Khaluf & Attinah & Alreesh & Al-Godn & Shanaah & All sites \\
\hline \multirow{2}{*}{ Gymnuridae } & 14.3 & 15.4 & $\underline{18.2}$ & $\underline{12.7}$ & $\underline{9.0}$ & 7.7 & $\underline{0.0}$ & $\underline{14.6}$ \\
\hline & 2.1 & 0.8 & 1.2 & 1.6 & $\overline{0.8}$ & $\overline{0.6}$ & $\overline{0.0}$ & 0.8 \\
\hline \multirow{2}{*}{ Ariidae } & $\underline{0.0}$ & $\underline{6.7}$ & $\underline{12.7}$ & $\underline{3.7}$ & $\underline{6.3}$ & $\underline{19.4}$ & $\underline{0.0}$ & $\underline{10.4}$ \\
\hline & $\overline{0.0}$ & $\overline{3.7}$ & $\overline{23.9}$ & $\overline{5.5}$ & $\overline{23.5}$ & $\overline{35.7}$ & $\overline{0.0}$ & $\overline{19.8}$ \\
\hline \multirow{2}{*}{ Dasyatidae } & $\underline{0.2}$ & $\underline{8.3}$ & $\underline{16.1}$ & $\underline{4.3}$ & $\underline{6.8}$ & $\underline{2.6}$ & $\underline{8.0}$ & $\underline{10.3}$ \\
\hline & 0.4 & 1.6 & 0.5 & 0.3 & 1.2 & 0.4 & 0.1 & 0.7 \\
\hline \multirow{2}{*}{ Antennariidae } & $\underline{0.6}$ & $\underline{28.4}$ & $\underline{4.0}$ & $\underline{25.5}$ & $\underline{0.0}$ & $\underline{0.0}$ & $\underline{0.0}$ & $\underline{10.2}$ \\
\hline & 0.2 & 19.3 & 10.9 & 34.7 & 0.0 & 0.0 & 0.0 & 7.2 \\
\hline \multirow{2}{*}{ Leiognathidae } & $\underline{0.4}$ & $\underline{4.2}$ & $\underline{6.0}$ & $\underline{1.4}$ & 21.4 & $\underline{15.9}$ & $\underline{69.3}$ & 9.2 \\
\hline & 1.8 & 22.2 & 21.8 & 3.8 & 42.9 & 35.8 & 91.5 & 36.0 \\
\hline \multirow{2}{*}{ Synodontidae } & $\underline{0.8}$ & 1.4 & $\underline{5.5}$ & $\underline{2.0}$ & $\underline{6.8}$ & $\underline{2.3}$ & $\underline{0.4}$ & $\underline{3.5}$ \\
\hline & $\overline{3.9}$ & $\overline{2.2}$ & $\overline{4.5}$ & $\overline{2.5}$ & $\overline{1.3}$ & $\overline{0.8}$ & $\overline{0.1}$ & $\overline{2.2}$ \\
\hline \multirow{2}{*}{ Sciaenidae } & $\underline{0.0}$ & 1.0 & 6.2 & 1.7 & $\underline{0.1}$ & 1.1 & $\underline{0.0}$ & $\underline{3.1}$ \\
\hline & 0.0 & 1.8 & 8.1 & 4.4 & 0.4 & 0.7 & 0.0 & 3.1 \\
\hline \multirow{2}{*}{ Tetraodontidae } & $\underline{31.3}$ & $\underline{1.6}$ & $\underline{1.0}$ & $\underline{5.2}$ & $\underline{2.8}$ & $\underline{1.8}$ & $\underline{0.5}$ & $\underline{3.0}$ \\
\hline & $\overline{1.4}$ & $\overline{2.4}$ & $\overline{1.3}$ & $\overline{3.1}$ & $\overline{1.1}$ & $\overline{1.2}$ & $\overline{0.0}$ & $\overline{1.3}$ \\
\hline \multirow{2}{*}{ Sillaginidae } & $\underline{14.1}$ & $\underline{2.4}$ & $\underline{0.1}$ & $\underline{0.1}$ & $\underline{9.3}$ & $\underline{6.3}$ & $\underline{2.6}$ & 2.9 \\
\hline & 0.2 & 6.6 & $\overline{0.1}$ & $\overline{0.3}$ & 3.1 & 3.6 & $\overline{0.8}$ & 2.5 \\
\hline \multirow{2}{*}{ Plotosidae } & $\underline{0.0}$ & $\underline{5.8}$ & $\underline{0.4}$ & $\underline{0.2}$ & $\underline{0.0}$ & $\underline{0.0}$ & $\underline{0.6}$ & $\underline{1.8}$ \\
\hline & $\overline{0.0}$ & $\overline{8.3}$ & $\overline{1.5}$ & $\overline{1.2}$ & $\overline{0.0}$ & $\overline{0.1}$ & $\overline{0.8}$ & 1.9 \\
\hline \multirow{2}{*}{ Other fishes } & $\underline{33.9}$ & $\underline{18.7}$ & $\underline{28.2}$ & $\underline{39.6}$ & $\underline{27.1}$ & $\underline{31.3}$ & $\underline{17.3}$ & $\underline{26.2}$ \\
\hline & 86.9 & 30.2 & 24.2 & 41.1 & 25.1 & 20.9 & 6.7 & 23.7 \\
\hline Unidentified crabs & 0.0 & 0.8 & 0.7 & 2.8 & 8.3 & 11.3 & 1.1 & 2.6 \\
\hline \multirow{2}{*}{ Sepiidae } & 4.3 & 5.2 & 0.9 & 0.8 & 2.0 & 0.3 & 0.3 & 2.2 \\
\hline & 3.1 & 0.9 & 2.0 & 1.6 & 0.7 & 0.2 & 0.0 & 1.0 \\
\hline
\end{tabular}

Values above the line are percentage in weight $[\mathrm{kg}]$, values under the line is percentage in numbers.

Top ten species of the bycatch in percentages of weight and number

Table 4 during the shrimp trawl survey 2013

\begin{tabular}{|c|c|c|c|c|c|c|c|c|}
\hline \multirow{2}{*}{ Species } & \multicolumn{8}{|c|}{ Site } \\
\hline & Ras Markaz & Duqm & Khaluf & Attinah & Alreesh & Al-Godn & Shanaah & All sites \\
\hline \multirow{2}{*}{ Gymnura poecilura } & $\underline{14.3}$ & $\underline{15.4}$ & $\underline{18.2}$ & 12.7 & $\underline{9.0}$ & $\underline{7.7}$ & $\underline{0.0}$ & $\underline{14.6}$ \\
\hline & 2.1 & 0.8 & 1.2 & 1.6 & 0.8 & 0.6 & 0.0 & 0.8 \\
\hline \multirow{2}{*}{ Antennarius coccineus } & $\underline{0.6}$ & $\underline{28.4}$ & $\underline{4.0}$ & $\underline{25.5}$ & $\underline{0.0}$ & $\underline{0.0}$ & $\underline{0.0}$ & $\underline{10.2}$ \\
\hline & 0.2 & 19.3 & 10.9 & 34.7 & 0.0 & $\overline{0.0}$ & $\overline{0.0}$ & $\overline{7.2}$ \\
\hline \multirow{2}{*}{ Plicofollis tenuispinis } & $\underline{0.0}$ & $\underline{2.7}$ & $\underline{10.8}$ & $\underline{0.0}$ & $\underline{4.4}$ & $\underline{13.3}$ & $\underline{0.0}$ & 7.4 \\
\hline & 0.0 & 2.2 & 12.2 & 0.0 & 15.4 & 24.5 & 0.0 & 12.0 \\
\hline \multirow{2}{*}{ Photopectoralis bindus } & 0.0 & $\underline{0.6}$ & $\underline{4.5}$ & $\underline{0.0}$ & $\underline{9.4}$ & $\underline{14.7}$ & $\underline{0.0}$ & $\underline{4.7}$ \\
\hline & 0.0 & 7.0 & 16.0 & 0.0 & 24.1 & 29.5 & 0.0 & 16.3 \\
\hline \multirow{2}{*}{ Saurida tumbil } & $\underline{0.1}$ & $\underline{1.3}$ & $\underline{4.6}$ & $\underline{2.0}$ & $\underline{4.3}$ & $\underline{1.6}$ & $\underline{0.1}$ & $\underline{2.8}$ \\
\hline & 0.4 & 2.1 & 3.9 & 2.5 & 0.8 & 0.4 & 0.1 & 1.8 \\
\hline \multirow{2}{*}{ Lagocephalus guentheri } & $\underline{31.3}$ & $\underline{1.3}$ & $\underline{0.9}$ & $\underline{5.2}$ & $\underline{0.7}$ & $\underline{1.3}$ & $\underline{0.0}$ & $\underline{2.7}$ \\
\hline & 1.4 & 0.7 & 1.2 & 3.1 & 0.6 & 0.9 & 0.0 & 0.9 \\
\hline \multirow{2}{*}{ Himantura uarnak } & $\underline{0.0}$ & $\underline{5.1}$ & $\underline{1.9}$ & $\underline{0.0}$ & $\underline{0.0}$ & $\underline{1.6}$ & $\underline{0.0}$ & $\underline{2.4}$ \\
\hline & 0.0 & 0.2 & 0.1 & 0.0 & 0.0 & 0.1 & 0.0 & 0.1 \\
\hline \multirow{2}{*}{ Sepia pharaonis } & $\underline{4.3}$ & $\underline{5.2}$ & $\underline{0.9}$ & $\underline{0.0}$ & $\underline{1.6}$ & $\underline{0.3}$ & $\underline{0.3}$ & $\underline{2.2}$ \\
\hline & 1.2 & 0.9 & 2.0 & 0.0 & 0.7 & 0.2 & 0.0 & 1.0 \\
\hline \multirow{2}{*}{ Leiognathus oblongus } & $\underline{0.0}$ & $\underline{0.0}$ & $\underline{0.4}$ & $\underline{0.0}$ & $\underline{0.8}$ & $\underline{0.1}$ & $\underline{60.2}$ & $\underline{2.0}$ \\
\hline & $\overline{0.0}$ & $\overline{0.1}$ & $\overline{1.9}$ & $\overline{0.0}$ & $\overline{1.9}$ & $\overline{0.3}$ & $\overline{87.8}$ & 11.9 \\
\hline \multirow{2}{*}{ Aurigequula fasciata } & 0.0 & 3.5 & 0.2 & 0.0 & 11.2 & 1.1 & 0.0 & 1.8 \\
\hline & 0.0 & 13.7 & 1.4 & 0.4 & 17.0 & 5.8 & 0.0 & 6.2 \\
\hline
\end{tabular}

Values above the line are percentage in weight $[\mathrm{kg}]$, values under the line is percentage in numbers. 
more frequently during southwest monsoon in June-July and from June to October, respectively. Among bony fishes, representatives of families Antennariidae occurred in larger quantities in February and May, Ariidae were more abundant between November and January, and Leiognathidae in April-May (Fig. 4A). Among ray species, Gymnuridae was found more frequently from November to January and Dasyatidae in February-April (Fig. 4B). During open 3-months shrimp-fishing season (September-November) in the bycatch by weight dominated sea catfishes (Ariidae), rays (Gymnuridae), and ponyfishes (Leiognathidae).

\section{DISCUSSION}

During the presently reported survey, the mean bycatch to shrimp catch ratio was estimated at $8.9: 1$. There are many estimates of the bycatch/shrimp ratio for international shrimp trawl fisheries. Our results correspond to the rate and composition of the bycatch in Kuwaiti shrimp fishery (ratio $13.7: 1$ ) in the Arabian Gulf (Chen et al. 2013) and Iranian shrimp fishery (ratio $7: 1$ ) in the Bushehr coastal waters of the northern Arabian (Persian) Gulf (Paighambari and Daliry 2012), although they used other types of trawls and had other target shrimp species. Kuwaiti and Iranian shrimp fisheries target three shrimp
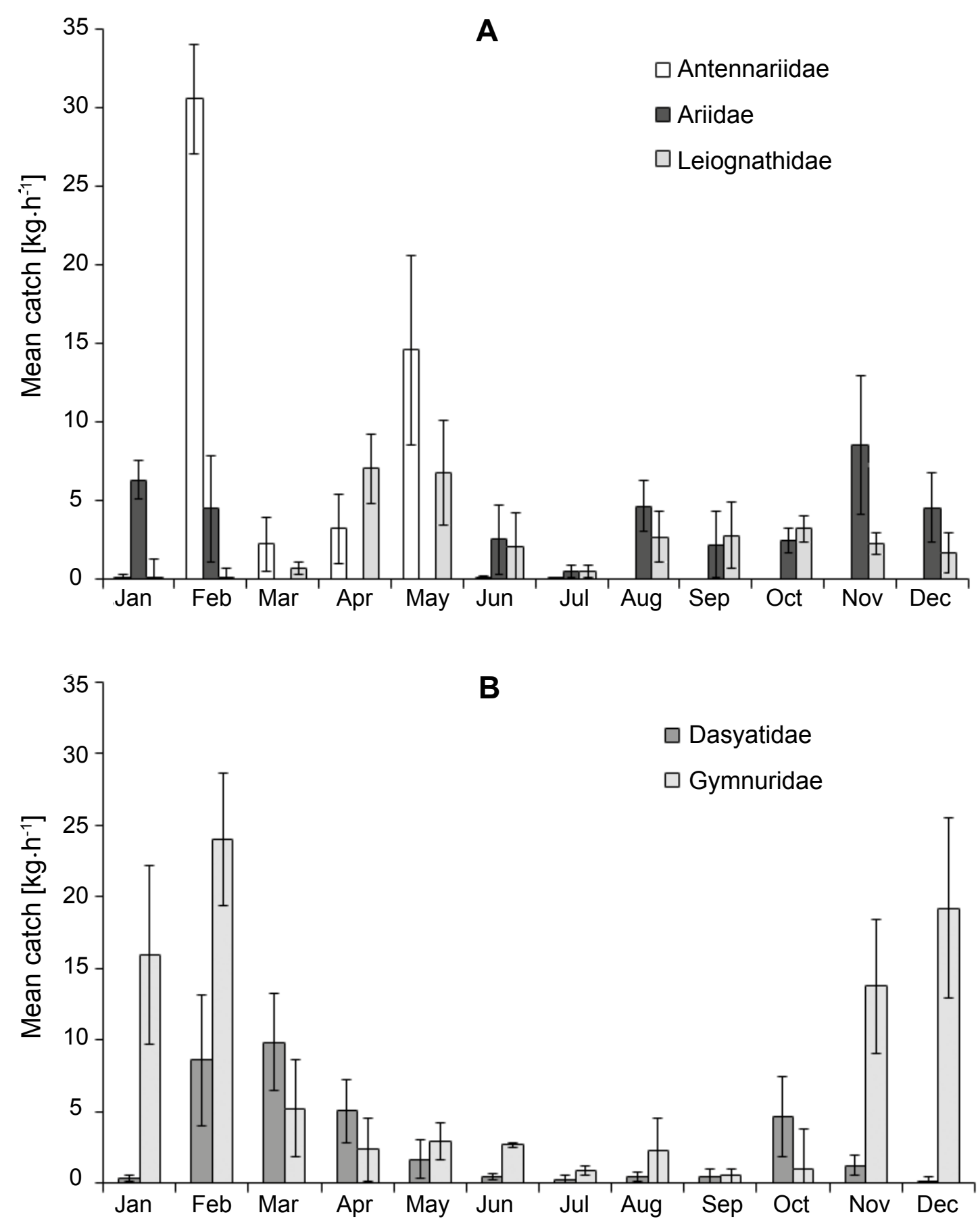

Fig. 4. Monthly changes of mean catches of the dominant families of bony fishes (A) and elasmobranchs (B) during 2013 
Values of indices of biological diversity in different sites (based on weight data)

\begin{tabular}{|c|c|c|c|c|c|c|c|}
\hline \multirow{2}{*}{ Species } & \multicolumn{7}{|c|}{ Site } \\
\hline & Ras Markaz & Duqm & Khaluf & Attinah & Alreesh & Al-Godn & Shanaah \\
\hline Shannon $H^{\prime}$ Log Base & 0.91 & 1.16 & 1.23 & 0.99 & 1.35 & 1.31 & 0.63 \\
\hline Simpsons Diversity $(1 / D)$ & 6.08 & 7.47 & 8.93 & 6.62 & 17.92 & 11.53 & 2.41 \\
\hline Margaleff & 54.78 & 40.14 & 39.35 & 71.82 & 54.81 & 46.63 & 62.51 \\
\hline Hill's & 29.26 & 66.87 & 86.71 & 38.43 & 127.29 & 111.87 & 11.84 \\
\hline
\end{tabular}

species: Penaeus semisulcatus, Metapenaeus affinis, Parapenaeopsis stylifera, and usually, more than $50 \%$ of catch was $P$. semisulcatus. The main target species in Oman is also $P$. semisulcatus, but two other shrimps are different (Penaeus indicus and Metapenaeus stebbingi).

These shrimp trawl fisheries are characterized not only by a great amount of bycatch biomass, but also by a high taxonomic diversity and similarity of marine animals. Specifically, Paighambari and Daliry (2012) found 114 species from 45 bony fish families, 13 species from 7 elasmobranch families, and 13 species from 13 invertebrate families. Chen et al. (2013) recorded 92 species of bony fishes, 6 species of sharks and rays, 3 species of cephalopods, and at least 10 species of crustaceans. We recorded 97 bony fish species from 36 families, 12 elasmobranch species from 7 families, and several species of cephalopods and crabs. Despite the high diversity these fisheries, usually very few species and families dominate in the bycatch. Paighambari and Daliry (2012) found in Iran predominance representatives of bony fish families Mullidae, Ariidae, Leiognathidae, Haemulidae, Clupeidae, rays Dasyatidae and Myliobatidae, and large biomass of crabs. Chen et al. (2013) reported that in the bycatch of Kuwaiti fishery the most dominant species were bony fishes Saurida tumbil (family Synodontidae), Nematalosa nasus (Bloch, 1795) (Clupeidae), Sphyraena flavicauda Rüppell, 1838 (Sphyraenidae), Acanthopagrus latus (Houttuyn, 1782) (Sparidae), Otolithes ruber (Bloch et Schneider, 1801) (Sciaenidae), and blue crab, Portunus pelagicus. During our study, we also found all these families and species (excluding S. flavicauda), however the bycatch structure was different. According to our data, the fish families that dominated in the bycatch in the Omani shrimp fishery in biomass, number of individuals, and frequency of occurrence were Ariidae, Antennariidae, Gymnuridae, Dasyatidae, and Leiognathidae. The most abundant species found were Gymnura poecilura, Himantura uarnak, Antennarius coccineus, Netuma thalassina, Plicofollis tenuispinis, and Photopectoralis bindus. The large number of species registered with, however, the numerical predominance of very few of them, was characteristic of the shrimp trawl bycatch in tropical waters (Clucas 1998).

Calculation of diversity indices showed that the diversity and similarity of the bycatch composition in various studied sites of Oman was very high. The cluster analysis indicated a greater similarity among sites from the nearby geographical areas, especially among central sites Attinah, Alreesh, and Al-Godn. The marginal sites showed the higher rate of the bycatch animals in weight (Ras Markaz, Duqm, Khaluf) and numbers (Shanaah). This finding indicated that Attinah, Alreesh, and Al-Godn (so-called Mahout region) is the best place for the shrimp trawl fishery from a resource conservation point of view, because it has the lowest rate of the bycatch species. The results of the presently reported study also showed that the period of the lowest bycatch rate was observed from June until September, which coincides with the southwest monsoon. However, during this monsoon season the water is very rough in the Arabian Sea and the shrimp trawl fishery is limited. The lower bycatch to shrimp catch ratio also was observed during pre-monsoon months (April-May), so it can be recommended to consider the shift of the current shrimp fishing season from September-November to March-May.

From the bony fishes and rays registered during the current study, the majority was demersal, living on muddy sand bottoms and in rocky reefs of shallow inshore waters and offshore banks. The predominance of rays, catfishes, frogfish, and ponyfishes in weight as well as in numbers may be attributed to the importance of these species for this geographical region and biotope. Pelagic species as representatives of family Clupeidae and Engraulidae constituted very small part of the catch.

Small-sized specimens and slow-moving animals represented the majority of fishes caught by the shrimp otter trawl. Species caught as a bycatch are mainly non-edible (frogfish) or with low economic value (catfishes, rays, ponyfishes, etc.). Economically valuable species, like carangids, clupeids, mugilids, mullids, and sparids were caught more often in juvenile stages, which threatens their population structure, and potential yields in artisanal fisheries targeting these species. Similarly, for the same reason, other species of fish, crabs, squids, and cuttlefish found in the bycatch may be threatened. It is worthy to note that fishers usually do not discard the bycatch back to the sea, but retain it on board and then use it for personal consumption, give it to helpers, or sell it on the local fish market. Although shrimp trawl fisheries provide social and economic benefits, they produce ecological problems and affect other fishery activities that need special consideration. It is possible that the heavy exploitation of shrimp would have led to a situation favourable for the thriving of the undesirable species, which occupy similar ecological niches in estuaries and coastal ecosystems. In addition, knowledge of the effect of trawl gear on the bottom living resources is important as many estuarine fish species de- 
pend on these organisms as food sources. Ali et al. (1993) found that crabs, shrimps, and molluscs are the major food items for the demersal fish assemblage in the Northwest Arabian Gulf.

The presently reported study is the first research of the bycatch level and species composition from shrimp trawl fisheries in Oman. However, much more work still needs to be done for better understanding what environmental factors (such as habitat parameters, temperature, salinity, depth, etc.) and biological factors (species composition, spawning, migrations) are the most important determinants of spatio-temporal changes of the bycatch structure and abundance. In addition, there has been a need to improve the fishing techniques and methods (boats, gears, mesh size, tow duration, etc.) to increase shrimp landings and reduce bycatch from such complex marine ecosystem.

The results from the study on the bycatch of the shrimp trawl fishery in Oman point out the necessity of management actions to maintain both a shrimp fishery industry and the ecosystem functioning that sustain it. Basically, it is necessary to develop Oman's policy on bycatch reduction not only for the shrimp fishery but also for other fishery activities.

\section{ACKNOWLEDGEMENTS}

The authors are very grateful to the Agriculture and Fisheries Development Fund of the Ministry of Agriculture and Fisheries Wealth, Sultanate of Oman, for financial support of the project. We are thankful to Al-Wusta Department of Fisheries Development, Muscat, Sultanate of Oman, for the logistic help, field trip arrangement and labour help during the survey in the region. We express our gratitude to our colleagues in the Marine Science and Fisheries Centre for their support in the identification of fish species. We also wish to thank all local fishers for their help during sampling operations.

\section{REFERENCES}

Al-Abdessalaam T.Z. 1995. Marine species of the Sultanate of Oman. An Identification Guide. Ministry of Agriculture and Fisheries, Sultanate of Oman, Publication No. 46/95.

Ali T.S., Mohamed A.R.M., Hussain N.A. 1993. Trophic interrelationships of demersal fish assemblage in the northwest Arabian Gulf, Iraq. Asian Fisheries Science 6 (3): 255-264.

Alverson D.L., Freeberg M.H., Murawski S.A., Pope J.G. 1994. A global assessment of fisheries bycatch and discards. FAO Fisheries technical paper No. 339. Rome, FAO.
Anonymous 2014. Fisheries statistics book 2013. General Directorate of Planning and Development. Fisheries Statistics Department. Ministry of Agriculture and Fisheries, Muscat, Oman.

Chen W., Almatar S., Alsaffar A., Yousef A.R. 2013. Retained and discarded bycatch from Kuwait's shrimp fishery. Aquatic Science and Technology 1 (1): 86-100. DOI: 10.5296/ast.v1i1.2778

Clucas I. 1998. Bycatch-is it a bonus from the sea? Infofish International 3: 24-28.

Fischer W., Bianchi G. (eds.) 1984. FAO species identification sheets for fishery purposes. Western Indian Ocean; (Fishing Area 51). Prepared and printed with the support of the Danish International Development Agency (DANIDA). Rome, Food and Agricultural Organization of the United Nations, Vols. 1-6.

Froese R., Pauly D. (eds.) 2015. FishBase. [version 04/2015] http://www.fishbase.org

Gibinkumar T.R., Sabu S., Pravin P., Boopendranath M.R. 2012. Bycatch characterization of shrimp trawl landings off southwest coast of India. Fishery Technology 49 (2): 132-140.

Kazemi S.H., Paighambari S.Y., Naderi, R.A. 2013. Species composition of trawl shrimp by-catch in the fishing grounds of northern Persian Gulf (Hormuzgan Province). World Journal of Fish and Marine Sciences 5 (5): 505-510.

DOI: 10.5829/idosi.wjfms.2013.05.05.73226

Kelleher K. 2005. Discards in the world's marine fisheries. FAO Fisheries Technical Paper 470. Rome, FAO. 2005.

Mahesh V., Benakappa S., Dineshbabu A.P., Anjanayappa H.N., Kumar Naik A.S., Vijaykumar M.E., Somashekara S.R., Kumar J. 2014. Finfish constituents of trawl low value by-catch off Mangalore. Journal of Experimental Zoology India 17 (2): 479-485.

Paighambari S.Y., Daliri M. 2012. The by-catch composition of shrimp trawl fisheries in Bushehr coastal waters, the northern Persian Gulf. Journal of the Persian Gulf (Marine Science) 3 (7): 27-36.

Randall J.E. 1995. Coastal fishes of Oman. Crowford House Publishing, Bathurst, Australia.

Shakir S., Bano A. 1999. Bycatch utilization in Pakistan. Pp. 179-190. In: Clucas I., Teutscher F. (eds.) Report and proceedings of FAO/DFID expert consultation on bycatch utilization in tropical fisheries, Beijing, China, 21-28 September 1998. FAO, Rome.

Received: 28 February 2015 Accepted: 16 September 2015 Published electronically: 30 September 2015 